\title{
Experimental Investigation on FRA Diagnosis of Transformer Faults
}

\author{
Takahiro Sano Member (Japan AE Power Systems Corporation, sano-takahiro@mb.jaeps.com) \\ Katsunori Miyagi Senior Member (Japan AE Power Systems Corporation, miyagi-katsunori@mb.jaeps.com)
}

Keywords: transformers, frequency response analysis, fault diagnosis, resonance

\section{Fault Detection Principles}

The impedance $Z$ of the transformer is a combined value mainly of the winding components (resistances, leakage reactances and capacitances) and the excitation components (conductance, susceptance and capacitance). The inductive $(L)$ and capacitive $(C)$ components are responsible for the transient and resonance, which occurs at a given frequency when the inductive reactance balances the capacitive reactance. The resonant frequency $f r$ is generally expressed by the Eq. (1).

$$
f r=\frac{1}{2 \pi \sqrt{L C}}
$$

\section{Test Circuits}

There are two ways of frequendy response analysis (FRA) measurement: one with the LV side of the transformer open-circuited, and the other with the LV side of transformer short-circuited. During an open-circuit test, the excitation components dominate the circuit. The other, during a short-circuit test, the phenomenon is reversed, the excitation components become negligible compared to the short-circuited winding resistance and leakage reactance.

[Case Study 1] Diagnosis on Lead Wire Breaks in Electrostatic Shields (Short-Circuit Test) An investigation was made on the assumption that the earth lead wire to the electrostatic shield was broken. Fig. 1 shows the FRA measurement results in case the disconnection of the lead wire of $\mathrm{W}$ phase only was simulated while the lead wires of $\mathrm{U}$ phase and $\mathrm{V}$ phase were connected. As a result, we could recognize the following: (1) The first resonant frequency of $\mathrm{W}$ phase is higher by approximately $26 \%$ than those of the other two phases. (2) The impedance of W phase at the first resonant frequency is higher than the impedance of the other two phases. (3) Differences in the characteristics of impedance are more significant at high frequencies than those at the first resonant frequency. (4) The characteristics of the impedance in a frequency range of $3 \mathrm{kHz}$ or below are same for the three phases. From EMTP simulation with a lumped parameter model of the transformer-windings in cases both with and without lead wire connection, we could find similar features in the actual FRA measurement and the simulation results.

[Case Study 2] Diagnosis of Double Core Earths (Open-Circuit Test) An investigation was made by comparing the case of normal core earth and the case of simulated double core earths. As a typical example of FRA measurement results, Fig. 2 shows the comparison of FRA waveforms from the HV side (U phase). The above comparison shows that differences in frequency response characteristics between normal core earth and double core earths are apparent in a lower frequency range. In addition, the frequency response characteristics were discovered to be such that the first resonance frequency was higher in the case of double core earths than normal core earth, and the impedance of the former is smaller than the latter. The reason for the above seems to be that

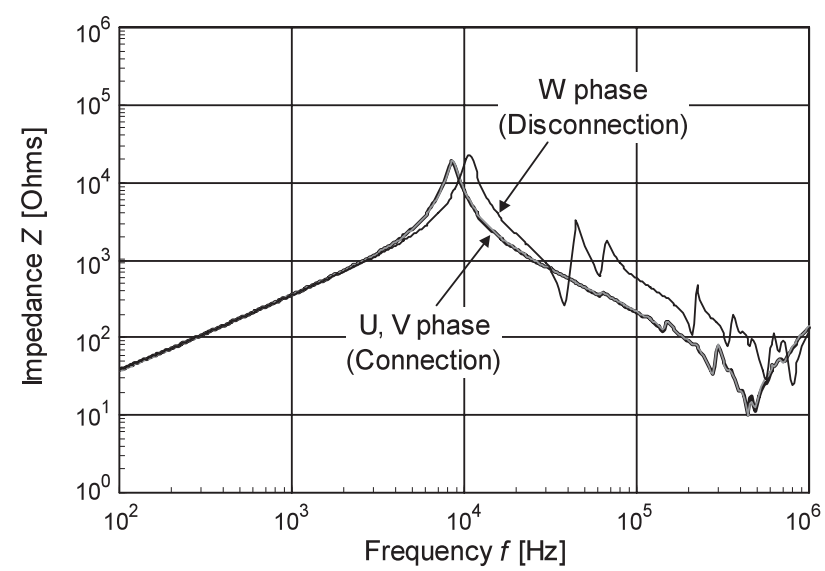

Fig. 1. FRA measurement result in the case study 1

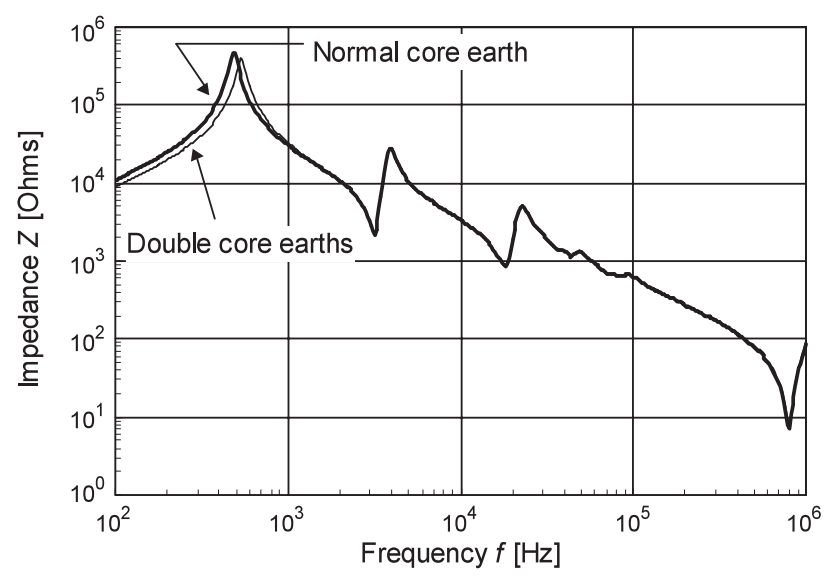

Fig. 2. FRA measurement result in the case study 2

the core was partially short-circuited owing to double earths, which lowered excitation susceptance and resulted in $f r$ increase according to the Eq. (1).

\section{Conclusion}

It could be seen that the lead wire broken in electrostatic shields and double core earths change frequency response characteristics respectively. These findings suggest the possibility of transformer diagnosis from outside by FRA measurements. It was confirmed that the location of damaged part could be estimated if examinations were performed by comparing the frequency response characteristics of three phases and by comparing between measurement and simulation characteristics.

For transformers evaluated as abnormal by DGA (Dissolved Gas Analysis) etc., the abnormal locations can be specified by FRA diagnosis before causing serious failures, and the transformer can be performed premeditated repair. 


\title{
Experimental Investigation on FRA Diagnosis of Transformer Faults
}

\author{
Takahiro Sano* Member \\ Katsunori Miyagi $^{* *}$ Senior Member
}

\begin{abstract}
The need for economical, reliable and effective delivery of electric power leads to the search for new, efficient and effective methods for diagnosing the high voltage equipments in the industries all over the world. As the average usage period of transformers increases, the necessity to know the internal condition of transformers is increasing. It is therefore critically important to establish monitoring and diagnostic techniques that can perform transformer condition assessment. Frequency response analysis, generally known as FRA, is one of the technologies to diagnose transformers.

Using case studies, this paper presents the effectiveness of FRA as measurements for detecting transformer faults. This paper introduces the fact that FRA waveforms have useful information about diagnosis of fault on winding shield and core earths, and that the condition outside transformers can affect frequency response characteristics. The FRA measurement results are further investigated through a simulation study using a computer model.
\end{abstract}

Keywords: transformers, frequency response analysis, fault diagnosis, resonance

\section{Introduction}

Power transformers being expensive items need to be carefully monitored throughout its operation. Currently, condition based maintenance and online monitoring are gaining importance. Frequency response analysis (FRA) has been found to be adequate for the evaluation of possible deformations or displacements of the transformer windings as technique to diagnose from the transformer outside ${ }^{(1)-(5)}$. While guidelines for the method of transformer diagnostic testing by FRA measurements are under consideration by CIGRE (SC A2) $\mathrm{WG}^{(6)}$, FRA measurement methods are yet to be firmly established.

In this paper, experiments were conducted on aged power transformers as test objects with simulated-faults. For example, the FRA measurements were able to be carried out with and without test bushings. Furthermore, the FRA characteristics about the cases of unusual core earth connection and the lead wire breaks in electrostatic shields were able to be obtained. It was found from the results of these experiments that the comparison of frequency response characteristics for individual phases was effective as diagnosis of transformers by FRA. The comparison to simulations suggested the possibility of pinpointing the fault parts by the FRA measurements. Therefore, it was discussed to be possible to prevent the serious failures by FRA diagnosis for fault locations in transformers. Furthermore, comparisons were carried out between measured and simulated FRA results.

\section{Principles and Measurement Example}

2.1 Fault Detection Principles The impedance $Z$ of the transformer is a combined value mainly of the winding

\footnotetext{
* Transformer Division, Japan AE Power Systems Corporation

585, Shimonakamizo Higashi Makado, Numazu 410-0865

** R \& D Division, Japan AE Power Systems Corporation

1-1-1, Kokubu-cho, Hitachi 316-8501
}

components (resistances, leakage reactances and capacitances) and the excitation components (conductance, susceptance and capacitance). The inductive $(L)$ and capacitive $(C)$ components are responsible for the transient and resonance, which occurs at a given frequency when the inductive reactance balances the capacitive reactance. The resonant frequency $f r$ is generally expressed by the Eq. (1).

$$
f r=\frac{1}{2 \pi \sqrt{L C}}
$$

There are two types of the resonances; parallel and series resonance. The parallel resonance is related to the parallel connection of the capacitance and inductance and has high impedance. On the contrary, the series resonance is related to the series connection, has very low impedance.

In addition, resonances can also occur locally between sections and turns of the windings. These resonances can be affected by changes of $L$ or $C$ that take place locally. FRA, which performs measurement over a wide frequency range, is capable of detecting many resonance points. Therefore, it is possible to estimate the locations of local changes that could not be detected through conventional diagnostic techniques.

2.2 Test Conditions and Circuits There are two methods of FRA measurement on a two-winding transformer from the HV side: one with the LV side of the transformer in Fig. 1(a) open-circuited, and the other with the LV side of transformer in Fig. 1(b) short-circuited ${ }^{(7)}$. During an opencircuit test, the excitation components and winding components are in series, so the excitation components dominate the circuit, and the winding resistance and leakage reactance are negligible at low frequency. The other, during a shortcircuit test, the phenomenon is reversed, with the opposite winding components in parallel. The excitation components become negligible compared to the short-circuited winding resistance and leakage reactance.

The measurement system is shown in Fig. 2. The input 


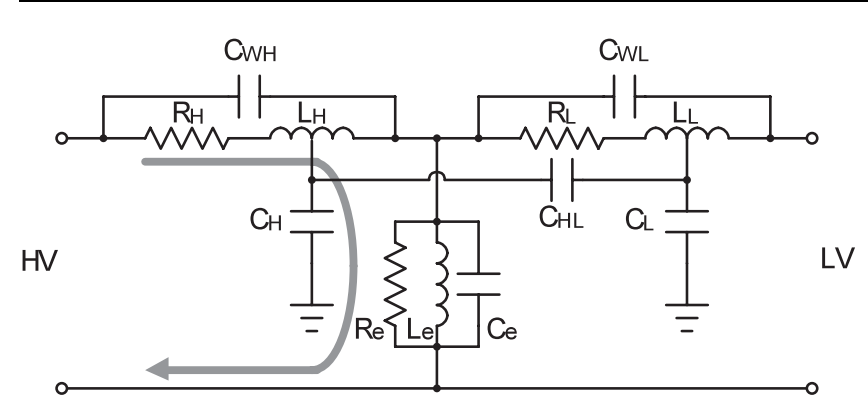

(a) Open-circuit test

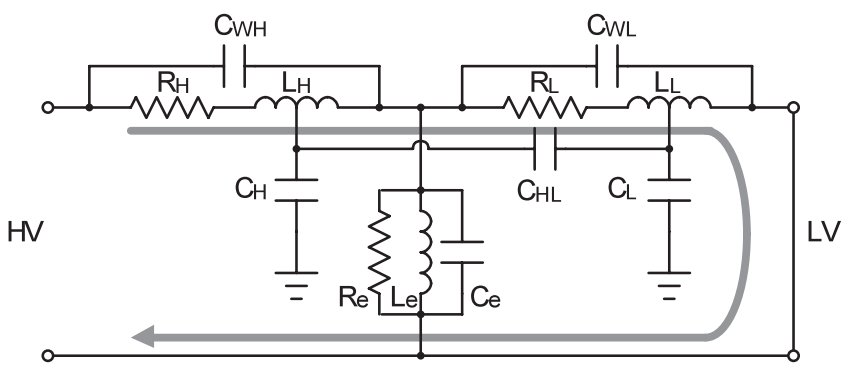

(b) Short-circuit test

$\mathrm{C}_{\mathrm{WH}}, \mathrm{C}_{\mathrm{WL}}$ : Inter-section capacitance $\quad \mathrm{C}_{\mathrm{H}}, \mathrm{C}_{\mathrm{L}} \quad$ : Capacitance to ground

$\mathrm{C}_{\mathrm{HL}}$ : Inter-winding capacitance $\mathrm{R}_{\mathrm{H}}, \mathrm{R}_{\mathrm{L}} \quad$ : Winding resistance

$\mathrm{L}_{\mathrm{H}}, \mathrm{L}_{\mathrm{L}} \quad$ : Inductance of windings $\quad \mathrm{Re}, \mathrm{Le}, \mathrm{Ce}$ : Excitation admittance

Fig. 1. Simplified equivalent circuits

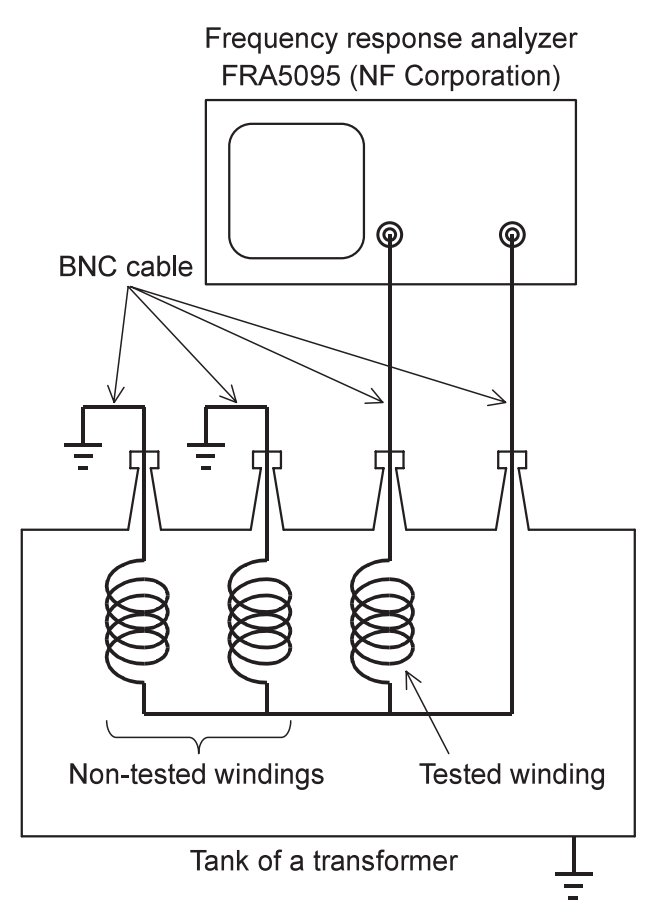

Fig. 2. FRA measurement system of a three-phase transformer

impedance of the measuring equipment is $1 \mathrm{M} \mathrm{ohm}$, and Table 1 shows the characteristics of the measurement cables. A sinusoidal wave which frequency is swept automatically from $100 \mathrm{~Hz}$ to $1 \mathrm{MHz}$ was applied to the high voltage terminal, and then the impedance $Z$ of the transformer was calculated from $V / I$. Where the sweep resolution was set to 400 steps/sweep (100 steps/div), and the applied voltage was set to 10 Vpeak.

2.3 FRA Measurement Example (Baseline Measurement) The waveforms by which we attempted to make
Table 1. Characteristics of measurement cable

\begin{tabular}{|c|c|}
\hline Name & 3D-2V BNC cable \\
\hline Characteristic impedance & $50 \Omega$ \\
\hline Length & $12 \mathrm{~m}$ \\
\hline
\end{tabular}

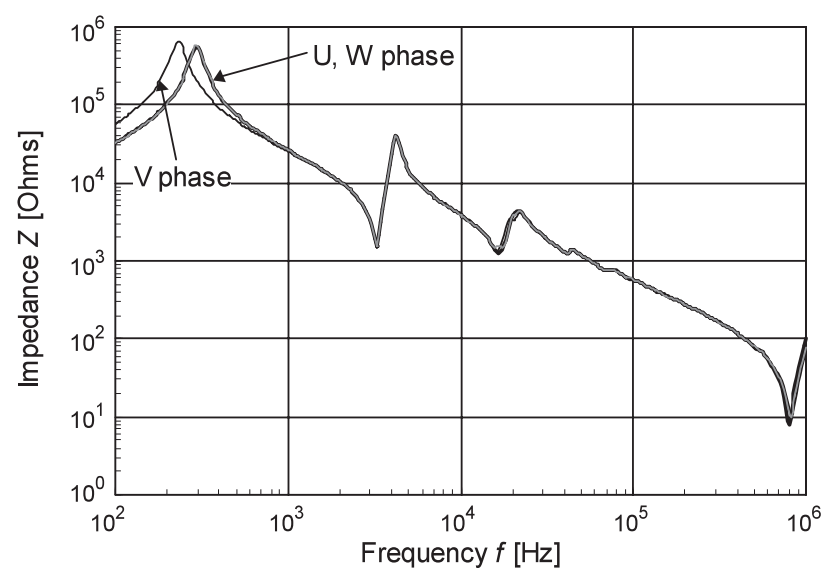

Fig. 3. FRA measurement example for the open-circuit test of a normal three-phase transformer $(220 \mathrm{kV}$, $120 \mathrm{MVA})$

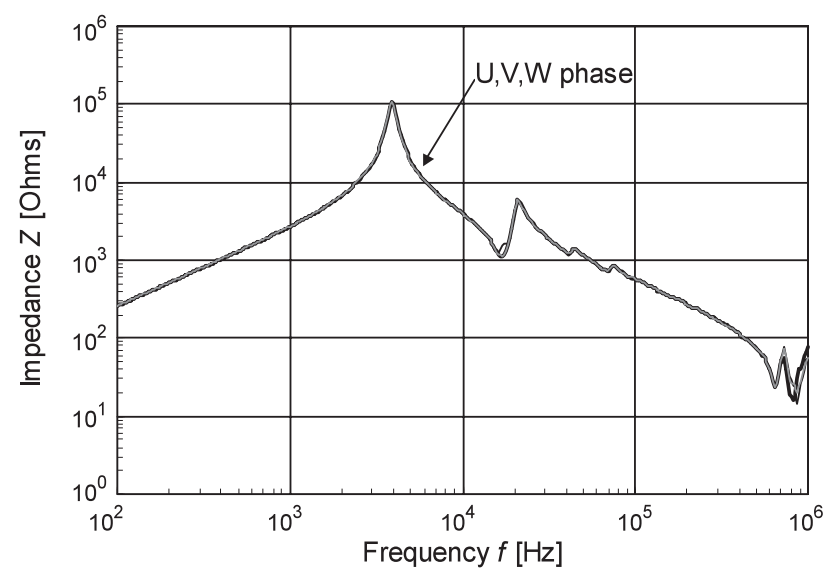

Fig. 4. FRA measurement example for the short-circuit test of a normal three-phase transformer $(220 \mathrm{kV}$, $120 \mathrm{MVA})$

comparison of individual phases with respect to FRA measurements on a normal three-phase transformer $(220 \mathrm{kV}$, 120 MVA) are shown in Figs. 3 and 4 in the case of open- and short-circuit respectively. The first peak frequency $(200 \mathrm{~Hz}$ to $300 \mathrm{~Hz}$ ) in Fig. 3 (open-circuit test) is lower than the first peak frequency (approx. $4 \mathrm{kHz}$ ) in Fig. 4 (short-circuit test). It can be supposed that compared to the leakage reactances of windings, the susceptance of excitation was so great that the frequency was dropped according to the Eq. (1).

From the comparison of frequency response characteristics in the cases of open- and short- circuit, the following two characteristics can be recognized:

(1) In the open-circuit test, over a range of $100 \mathrm{~Hz}$ to $1 \mathrm{kHz}$, the characteristics of the impedance of $\mathrm{V}$ phase are shifted to lower frequencies compared to those of the other phases $(\mathrm{U}, \mathrm{W})$.

(2) In the short-circuit test, the waveforms of the three phases were nearly same. 


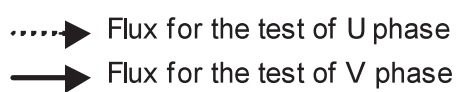

(Flux for the test of $\mathrm{W}$ phase is as symmetrical as U phase.)

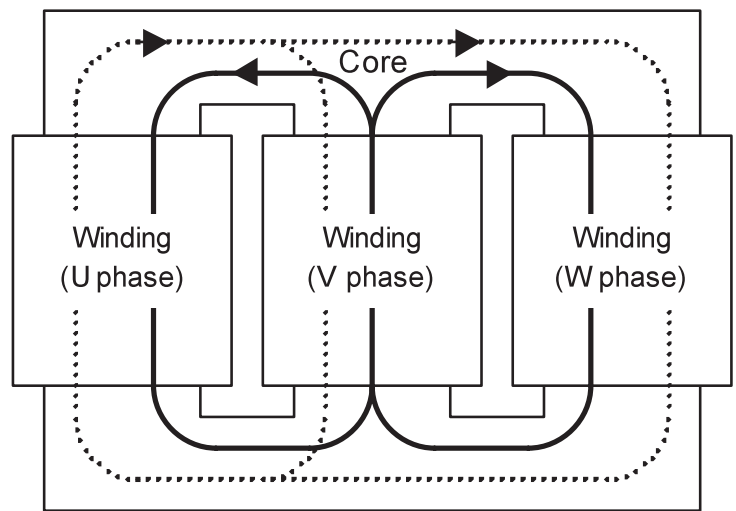

Fig. 5. The flow of flux in the case of the open-circuit test

As shown in Fig. 5, for the core structure, V phase is located in the center of the three-leg core and its magnetic path is shorter than the paths of the other phases. As the susceptance value, expressed as " $L$ " in the Eq. (1), is proportional to the length of a magnetic path, the susceptance value of $\mathrm{V}$ phase becomes smaller than the values of $U$ and $\mathrm{W}$ phases. In consequence, the resonant frequency $f r$ was shifted to the lower side as shown in the characteristics described in (1).

The winding structure of each phase was configured on the same axial symmetry, and the parameter values for the winding that could affect frequency response characteristics were nearly same in the three phases. In addition, they were free from the influence of the above-mentioned magnetic circuit. It can be judged that the feature described in (2) was obtained.

As shown above, even for a normal transformer, the FRA waveforms show the different characteristics because of the difference in the structures of individual phases in the opencircuit test, unlike the short-circuit test.

2.4 Influence of Factors Outside the Transformer (Short-Circuit Test) FRA measurements aim to diagnose damage to the transformer from outside by detecting a few differences in the waveforms that show frequency response characteristics. Should these differences be affected by external condition, fault diagnosis could not be performed with accuracy. The external condition includes, for example, measurement cables, power cables and bushings.

To investigate the influence of external factors, measurements were made on a normal three-phase power transformer ( $110 \mathrm{kV}, 40 \mathrm{MVA})$ with and without test bushings. FRA measurements at the factory test were made assuming the case with test bushings, and FRA measurements at the on-site test were made assuming the case without test bushings. These measurements were made through the short-circuit test, and the FRA measurement results are shown in Fig. 6. It was found from these results that the resonant frequency was reduced by approximately $7 \%$ in the case with test bushings compared to the resonant frequency in the case without test bushings.

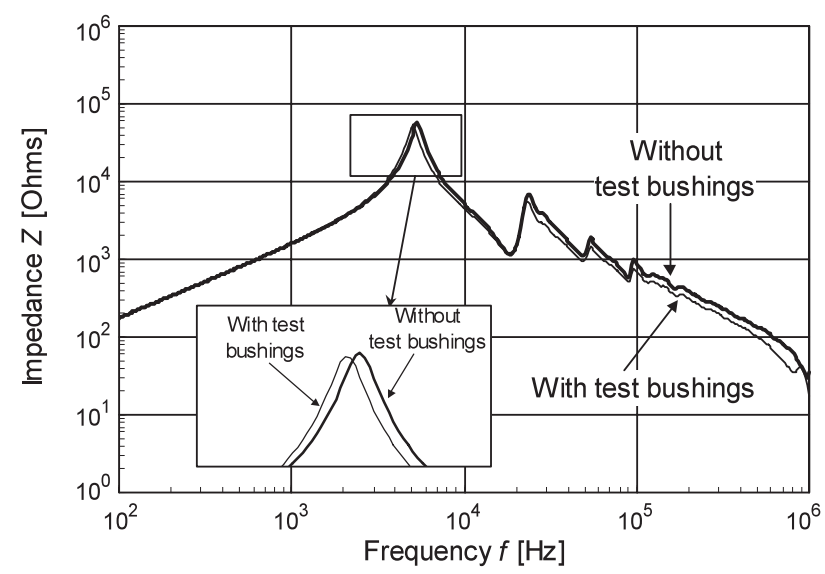

Fig. 6. FRA measurement result for the short-circuit test of a three-phase transformer $(110 \mathrm{kV}, 40 \mathrm{MVA})$

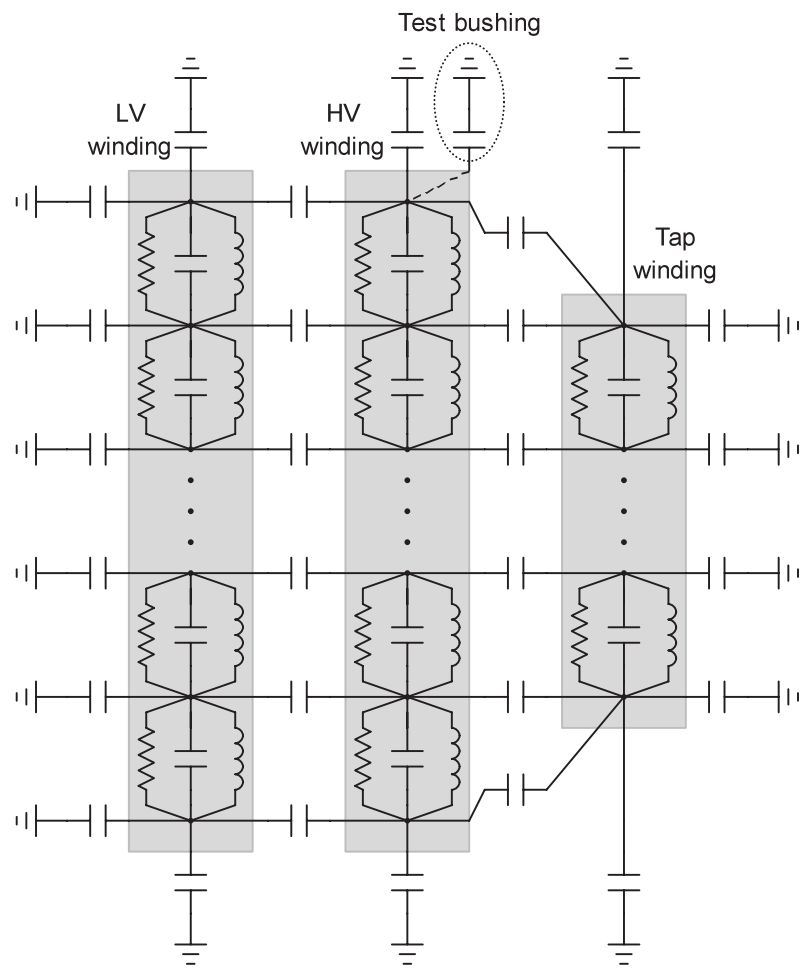

Fig. 7. Equivalent circuit of two-winding transformer windings for EMTP (single phase model)

For EMTP models with a lumped parameter model of the transformer-windings shown in Fig. 7 simulated in cases both with and without test bushings, Fig. 8 shows the results of respective calculations obtained using the "Frequency Scan" function of EMTP. From the above, we could find similar features in the actual FRA measurement and the simulation results.

Supposedly, these differences of the resonant frequency were caused by the capacitance of test bushings added to the capacitance to ground of the transformer, whereby $f r$ was reduced according to the Eq. (1). Table 2 shows the comparison with respect to capacitance to ground of the transformer in cases with and without test bushings. In the case with test bushings, from the Eq. (1), fr decreases in proportion to $1 / \sqrt{C}$ by approximately $8 \%$. This value is nearly same to the value obtained from the results of the FRA measurement, 


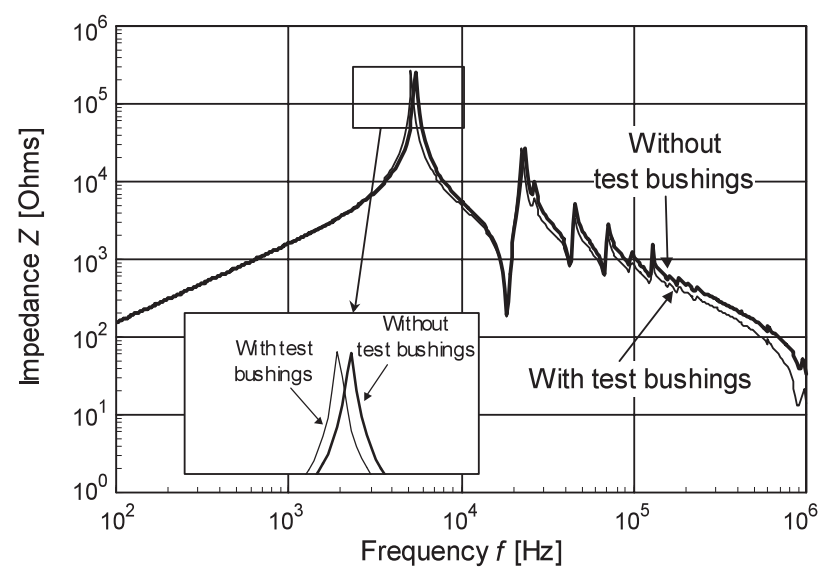

Fig. 8. FRA simulation result for the short-circuit test of a three-phase transformer ( $110 \mathrm{kV}, 40 \mathrm{MVA})$

Table 2. Capacitance to ground of the transformer (measurement result)

\begin{tabular}{|c|c|}
\hline Condition & Capacitance to ground \\
\hline With test bushings & $4100 \mathrm{pF} /$ phase \\
\hline Without test bushings & $3500 \mathrm{pF} /$ phase \\
\hline
\end{tabular}

so the differences in the above results are found to be attributable to the existence of test bushings only. As is presented above, because frequency response characteristics could be affected by the condition outside the transformer, much precaution is required when performing measurement.

\section{Diagnosing Faults (Case Studies)}

3.1 Case 1: Diagnosis on Lead Wire Breaks in Electrostatic Shields (Short-Circuit Test) Transformers, in which electrostatic shields were installed to prevent overvoltage between HV winding and tap winding when lightning surges strike the transformers, have been often manufactured $^{(8)}$. It is reported that electrostatic shields may be damaged by aged deterioration ${ }^{(9)}$. Even if a lead wire in an electrostatic shield breaks, the damage will not cause a failure in the transformer immediately, because it is not the main conducting circuits in the transformer. However, if the damage remains for a long duration, serious failures may occur owing to electric discharge and overheating around the lead wire in the transformer.

We selected a three-phase power transformer $(147 \mathrm{kV}$, $150 \mathrm{MVA}$ ) as a model for the diagnosis of lead wire breaks in electrostatic shields. This transformer is characterized by the electrostatic shield with ground potential installed between $\mathrm{HV}$ winding and tap winding.

Here, an investigation was made on the assumption that the earth lead wire to the electrostatic shield was broken shown in Fig. 9. Because frequency response characteristics about windings can be diagnosed through the short-circuit test, comparison and investigation of frequency response characteristics were performed between normal connection of the lead wire and simulated disconnection of the lead wire.

Fig. 10 shows the FRA measurement results in case the disconnection of the lead wire of $\mathrm{W}$ phase only was simulated while the lead wires of $U$ phase and $\mathrm{V}$ phase were connected. As a result, we could recognize the following:

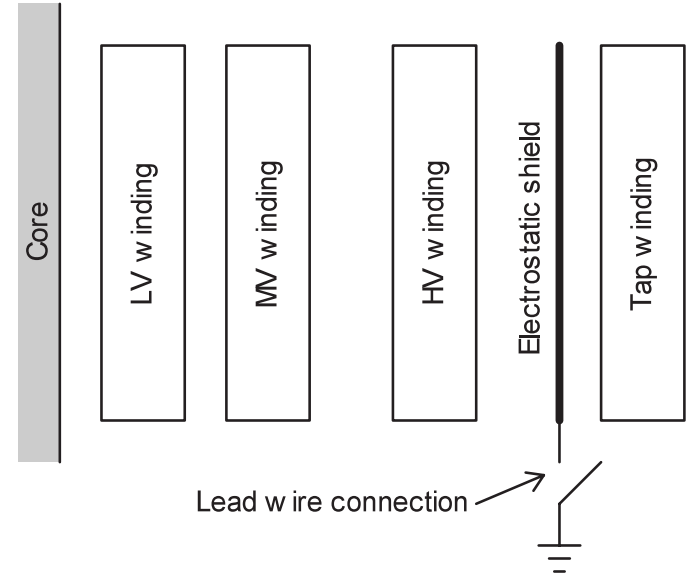

Fig. 9. Circuit condition for lead wire breaks model

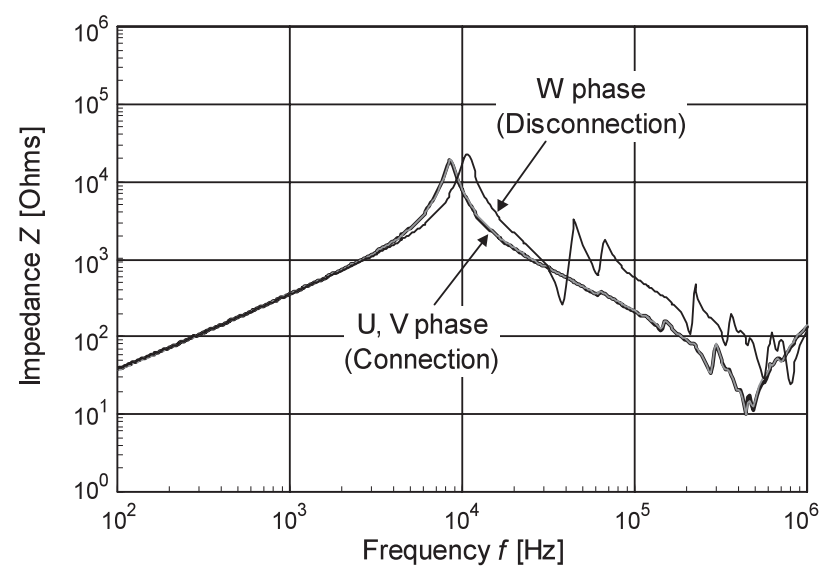

Fig. 10. FRA measurement result for the short-circuit test of a three-phase transformer $(147 \mathrm{kV}, 150 \mathrm{MVA})$

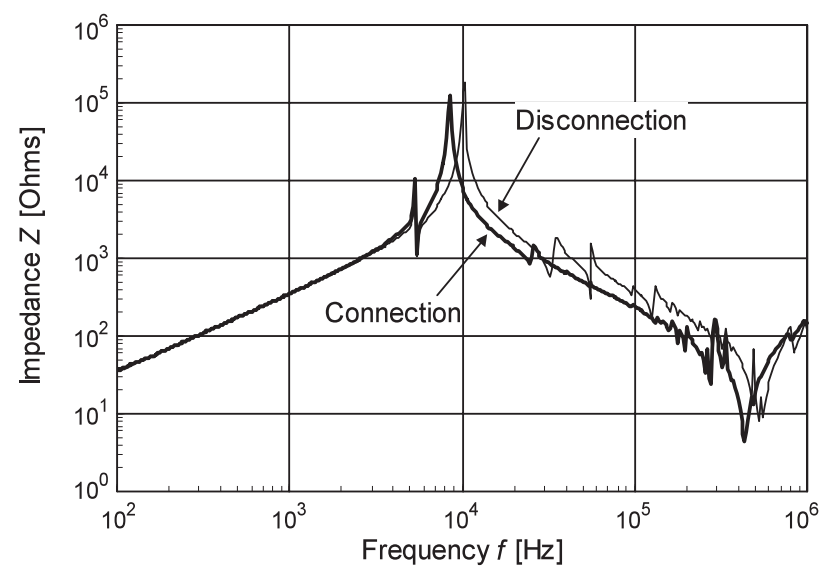

Fig. 11. FRA simulation result for the short-circuit test of a three-phase transformer $(147 \mathrm{kV}, 150 \mathrm{MVA})$

(1) The first resonant frequency of $\mathrm{W}$ phase is higher by approximately $26 \%$ than those of the other two phases.

(2) The impedance of $\mathrm{W}$ phase at the first resonant frequency is higher than the impedance of the other two phases.

(3) Differences in the characteristics of impedance are more significant at high frequencies than those at the first resonant frequency.

(4) The characteristics of the impedance in a frequency range of $3 \mathrm{kHz}$ or below are same for the three phases.

In addition, Fig. 11 shows the results of EMTP simulation 


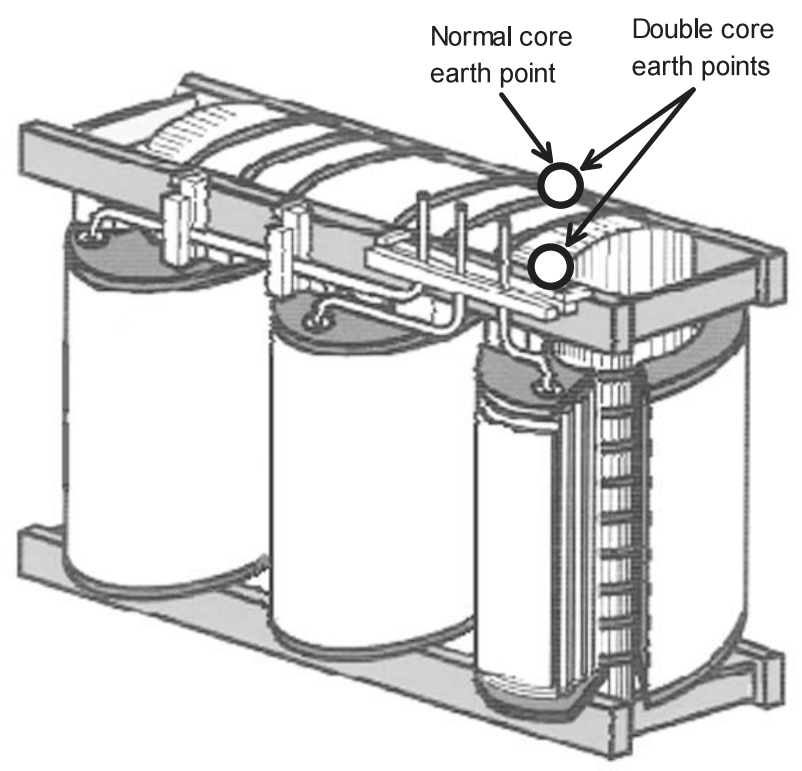

Fig. 12. Experimental core earth points for double core earths model

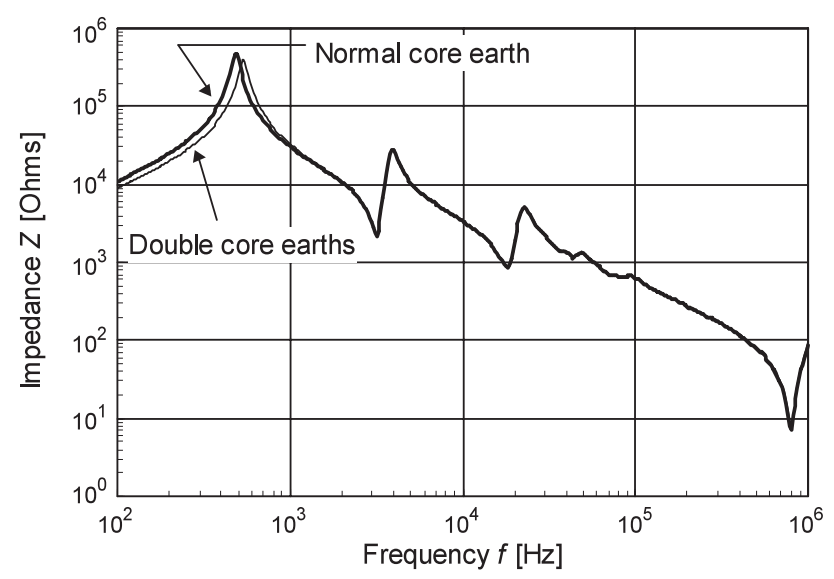

Fig. 13. FRA measurement result for the open-circuit test of a three-phase transformer $(230 \mathrm{kV}, 150 \mathrm{MVA})$

perfomed in cases with and without lead wire connection. We obtained the characteristics similar to those obtained through actual measurements.

In Fig. 11, resonance points at about $5 \mathrm{kHz}$ which do not exist in Fig. 10 are detected. There are many different points between measurement and simulation result including these resonance points. However, their causes still can not be evaluated well.

If the applicability of the simulation model can be confirmed, it will be possible, for example, to simulate frequency response characteristics by assuming the damaged part - the test technique that can be used for diagnosis such as identification of damaged part through comparison to FRA measurement results.

3.2 Case 2: Diagnosis of Double Core Earths (OpenCircuit Test) If the internal structure materials of the transformer including the core are multiple-earthed, local overheating can occur owing to circulating currents. Although it is rare that this causes a failure, dissolved gas in oil may be generated by the overheating.

We selected a three-phase power transformer $(230 \mathrm{kV}$,
150 MVA) to be used in the case study on double core earths. Frequency response characteristics about the core can be tested through the open-circuit test.

Here, an investigation was made by comparing the case of normal core earth and the case of simulated double core earths. The core earth points are shown in Fig. 12. As a typical example of FRA measurement results, Fig. 13 shows the comparison of the FRA waveforms from the HV side ( $\mathrm{U}$ phase). The above comparison shows that differences in frequency response characteristics between normal core earth and double core earths are apparent in a lower frequency range $(100 \mathrm{~Hz}$ to $1 \mathrm{kHz})$, whereas there are no significant differences in other frequency range $(1 \mathrm{kHz}$ to $1 \mathrm{MHz})$.

In addition, the frequency response characteristics were discovered to be such that the first resonance frequency was higher in the case of double core earths than normal core earth, and the impedance of the former is smaller than the latter. The reason for the above seems to be that the core was partially short-circuited owing to double earths, which lowered excitation inductance and resulted in fr increase according to the Eq. (1).

\section{Conclusion}

It could be seen that if the lead wire of the electrostatic shield was broken, the capacitance between HV winding and tap winding changed and caused frequency response characteristics to change significantly. It could also be seen that if the transformer core was double-earthed, excitation inductance changed, causing frequency response characteristics to change accordingly.

These findings suggest the possibility of transformer diagnosis from outside by FRA measurements. In addition, the influence of the conditions outside transformers could also been recognized over frequency response characteristics, so it is necessary to consider their influence at the time of diagnosis.

It was found that the characteristics similar to those of actually measured FRA waveforms could be obtained also in EMTP simulations of the transformer-windings. Particularly, it was confirmed that the location of damaged part could be estimated if examinations were performed by comparing the frequency response characteristics of three phases and by comparing between measurement and simulation characteristics.

For transformers evaluated as abnormal by DGA (Dissolved Gas Analysis) etc., the abnormal locations can be specified by FRA diagnosis before causing serious failures, and the transformer can be performed premeditated repair. It is reported that the transformer can be repaired premeditatedly without stopping power supply because the lead wire breaks of electrostatic shields were confirmed by FRA diagnosis ${ }^{(10)}$.

\section{Future Subject}

Should the capacitance or inductance inside the transformer change partially, changes to frequency response characteristics could become very small. The overall feature of the frequency response characteristics from these measurements and simulations are in agreement, whereas there can be found many differences in detailed parts. It will be necessary 
in the future to establish a simulation technique that can cope with a few changes.

FRA measurement helps to obtain impedance characteristics over a range from high to low frequencies with relative ease. However, there is influence from the measurement system in a high frequency range, so precaution is required at the time of actual measurement. In the present study, it was confirmed that the differences of frequency response characteristics became apparent depending on cases with and without test bushings. It will be therefore necessary in the future to determine other parameters of the measurement system that may affect frequency response characteristics and to establish a measurement technique for minimizing the influence from the measurement system.

(Manuscript received Aug. 25, 2006,

revised April 2, 2007)

\section{References}

( 1 ) E.P. Dick and C.C. Erven: "Transformer diagnostic testing by frequency response analysis", IEEE Trans. PAS, Vol.PAS-97, No.6, pp.2144-2153 (1978)

( 2 ) D. Oshima and E. Ozaki: "Investigation of the failure diagnostic method of the transformer by FRA", Proc. of 2001 Annual Conference of Power \& Energy Society, IEE Japan, No.326 (2001) (in Japanese)

( 3 ) J.A.S.B. Jayasinghe, Z.D. Wang, A.W. Darwin, and P.N. Jarman: "Practical Issues in Making FRA Measurements on Power Transformers", Proc. of 14th ISH, G-013 (2005)

( 4 ) K. Miyagi and T. Sano: "Examination on Diagnostic Method of Transformer by FRA", 2006 National Convention Record IEE Japan, No.5-203 (2006) (in Japanese)

( 5 ) K. Miyagi and T. Sano: "Examination on Diagnostic Method of Transformer by FRA (Part 2)", Proc. of 2006 Annual Conference of Power \& Energy Society, IEE Japan, No.258 (2006) (in Japanese)

( 6 ) CIGRE WG A2.26 Report: "Mechanical condition assessment of transformer windings using Frequency Response Analysis (FRA)", ELECTRA, No.228 (2006)
( 7 ) M. de Nigris, R. Passaglia, R. Berti, L. Bergonzi, and R. Maggi: "Application of modern techniques for the condition assessment of power transformers", CIGRE Session 2004, A2-207 (2004)

( 8 ) L.F. Blume, A. Boyajian, G. Camilli, T.C. Lennox, S. Minneci, and V.M Montsinger: Transformer Engineering, pp.455-457, Corona Publishing Co., Ltd., Tokyo (1966) (in Japanese)

(9) Electric Technology Research Association: "Advanced maintenance technology against deterioration of metal enclosed substation equipment", ETRA, No.61, Vol.3, pp.151-153 (2006) (in Japanese)

(10) T. Wada, S. Nomoto, T. Shimonosono, Y. Ogawa, and T. Sano: "One example of improvement in diagnosis accuracy for large capacity oil-immersed transformer by using Frequency Response Analysis (FRA) method", Proc. of 2006 Annual Conference of Power \& Energy Society, IEE Japan, No.259 (2006) (in Japanese)

Takahiro Sano (Member) was born in Shizuoka, Japan, on July 30,

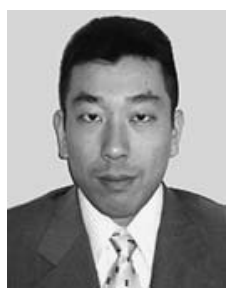
1974. He received B.E. degree in electrical engineering from Shizuoka University, Japan, in 1997. He worked for 5 years in Transformer Factory at Meidensha Corporation. In 2002, he joined Japan AE Power Systems Corporation, and is currently engaged in the design business of transformers.

Katsunori Miyagi (Senior Member) was born in Hokkaido, Japan, on

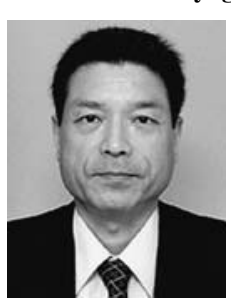
February 11, 1957. He received B.E., M.E. and Ph.D. degrees from Muroran Institute of Technology, Japan, in 1980, 1982 and 2000, respectively. He worked for 20 years in Transformer Factory at Meidensha Corporation. In 2002, he joined Japan AE Power Systems Corporation, where he is a manager of the Research \& Development Division. Dr. Miyagi has engaged in research on insulation technology of power transformers and measurement of high voltage. 